\title{
Rapid Short MRI Sequence Useful in Eliminating Stroke Mimics Among Acute Stroke Patients Considered for Intravenous Thrombolysis
}

Stephanie Paolini, Joselyn Burdine, Michael Verenes, James Webster, Theodore Faber, Cole Blease Graham and Souvik Sen*

Department of Neurology, University of South Carolina School of Medicine, Columbia, South Carolina, USA

\begin{abstract}
Background: Acute stroke teams are challenged by IV-tPA decision making in patients with acute neurological symptoms when the diagnosis is unclear. The purpose of this study was to evaluate the ability of the rapid Brain Attack Team (BAT) MRI in selecting patients for IV-tPA administration who present acutely to the emergency room with strokelike symptoms and an unclear diagnosis.

Methods: Consecutive patients were identified who presented within 4.5 hours of onset of stroke-like symptoms and considered for treatment with IV-tPA. When the diagnosis was not clear, a 9-minute BAT MRI was obtained. Stroke risk factors and $\mathrm{NIH}$ stroke scale obtained on presentation were compared between patients in whom BAT MRI was obtained and those in whom BAT MRI was not obtained. Similarly, comparisons were made between patients in whom BAT MRI detected abnormalities and those in whom BAT MRI did not detect abnormalities. BAT MRIs were analyzed to determine if radiological findings impacted clinical management and discharge diagnosis.

Results: In a 30-month period, 432 patients presenting with acute stroke-like symptoms were identified. Of these patients, 82 received BAT MRI. Patients receiving BAT MRI were younger, more likely to be smokers, and less likely to be selected for IV-tPA administration compared to those in whom a more definitive diagnosis of stroke precluded a BAT MRI. Of the 82 BAT MRIs, 25 were read as positive for acute ischemia. The patients with acute ischemia on BAT MRI were older, more likely to be males, have a history of hypercholesterolemia and atrial fibrillation, and more likely to be selected for IV-tPA administration compared to those with a negative BAT MRI. Of the 57 BAT MRIs read as negative for acute ischemia or hemorrhage, discharge diagnoses included TIA, MRI negative stroke, conversion/functional disorder, and multiple other illnesses.
\end{abstract}

Conclusion: In patients with acute stroke-like symptoms, BAT MRI may be used to confirm acute ischemic stroke, exclude stroke mimics, and assess candidacy for IV-tPA.

Keywords: Acute stroke imaging; Ultra rapid MRI; Thrombolysis

\section{Introduction}

Rapid diagnostic evaluation is essential to the effective treatment of acute ischemic stroke (AIS). Intravenous tissue plasminogen activator (IV-tPA) has the potential to minimize or eliminate long term disability of a patient who has suffered an ischemic stroke [1]. IV-tPA, however, is only FDA approved for administration within three hours of stroke onset [2]. Alternately, IV-tPA may be used off-label up to four and a half hours from stroke symptom onset [3]. In either case, time is critical for stroke patients and diagnostic imaging must be performed rapidly. Head CT can be performed quickly and is the standard of care when patients present acutely with symptoms suspicious for AIS. This mode of imaging, however, is not sensitive in detecting AIS and is primarily used to eliminate the possibility of a hemorrhage [4]. The use of CT alone in patients presenting with acute stroke-like symptoms can create an opportunity for stroke misdiagnosis to occur [5]. Stroke misdiagnosis may occur when a patient presents with a stroke mimic, which may be identified in up to 30 percent of patients who present with acute onset of neurological symptoms [6]. Although studies show that IV-tPA may be administered relatively safely in stroke mimics $[5,7]$, this treatment exposes the healthcare system to the unnecessary cost of tPA, hospitalization, and post-IV-tPA monitoring, typically in an intensive care unit [8]. Intravenous thrombolytic therapy has been shown to be safe and cost-effective for the treatment of AIS $[9,10]$. Although a recent study has shown that it is safe to administer IV-tPA to stroke mimics [11], impact of this on the health-care system remains unknown.

Since MRI has the capability to detect AIS and to effectively exclude stroke mimics, it is a valuable tool in the management of AIS.
Although MRI is the most sensitive test for AIS [12], the time required for a comprehensive brain MRI may delay treatment to stroke patients or put the patient outside the therapeutic window for IV-tPA. Delays in IV-tPA therapy should be minimized, as patients receiving the treatment earlier tend to have more favorable outcomes [13]. Recent studies have demonstrated the value of shorter MRI protocols in acute stroke [14-17]. To counter the delay created by comprehensive MRI, a rapid MRI sequence performed in less than 10 minutes called the Brain Attack Team MRI (BAT MRI) has been implemented at University of South Carolina-Palmetto Health Stroke Center. The purpose of this study was to evaluate the ability of the BAT MRI as a clinical tool to select patients for acute stroke treatment (specifically IV-tPA) who present acutely with questionable or inconsistent strokelike symptoms, such as altered mental status or seizure at onset of symptoms.

${ }^{*}$ Corresponding author: Souvik Sen, Professor and Chair, Department of Neurology, University of South Carolina School of Medicine, 8 Medical Park, Suite 420 Columbia, South Carolina 29203, USA, Tel: 803-545-6050/6073; Fax: 803545-6051; E-mail: Souvik.Sen@uscmed.sc.edu

Received September 20, 2013; Accepted October 21, 2013; Published October 24, 2013

Citation: Paolini S, Burdine J, Verenes M, Webster J, Faber T, et al. (2013) Rapid Short MRI Sequence Useful in Eliminating Stroke Mimics Among Acute Stroke Patients Considered for Intravenous Thrombolysis. J Neurol Disord 1: 137 doi:10.4172/2329-6895.1000137

Copyright: ( 2013 Paolini S, et al. This is an open-access article distributed under the terms of the Creative Commons Attribution License, which permits unrestricted use, distribution, and reproduction in any medium, provided the original author and source are credited. 
Citation: Paolini S, Burdine J, Verenes M, Webster J, Faber T, et al. (2013) Rapid Short MRI Sequence Useful in Eliminating Stroke Mimics Among Acute Stroke Patients Considered for Intravenous Thrombolysis. J Neurol Disord 1: 137. doi:10.4172/2329-6895.1000137

Page 2 of 5

\section{Methods and Materials}

The study was approved by the Institutional Review Board. A retrospective chart review was performed in consecutive patients who presented within 4.5 hours of onset of stroke-like symptoms and a code stroke BAT alert was activated. These patients were assessed by a neurologist member of the BAT, for a clear diagnosis of stroke vs. an uncertain diagnosis. The decision regarding the diagnosis was made on a case by case basis. Patients were assessed for stroke symptomatology, stroke risk factors, and stroke severity using NIH stroke scale (NIHSS). Patients in whom the stroke diagnosis was uncertain underwent a BAT MRI obtained rapidly by a pre-determined process implementation. These included patients with acute onset neurological signs and symptoms in whom a stroke mimic was suspected [6]. The predetermined process implementation included adding MRI technicians to the code stroke BAT page, a stat orderable called BAT MRI on the computerized physician order entry, the nurse filling out the MRI screening sheet, dedicated transport to take the patient to the MRI machine and the stroke physician available at the MRI console to read the BAT MRI on completion. The data collected by chart abstraction included stroke risk factors, information related to door-to-needle time (DTN) for IV-tPA administration, and discharge diagnoses. Patients in whom a BAT MRI was obtained were compared with patients in whom the clinical diagnosis of AIS was clear and a BAT MRI was not required to make an IV-tPA administration decision.

The BAT MRIs were performed on a Siemens 1.5 T (Symphony Tim $\mathrm{B} 17)$ or GE $1.5 \mathrm{~T}$ (Discovery 450) machine. The sequences included diffusion weighted image (DWI), T2 weighted image gradient echo imaging (GRE), T2 weighted image axial (T2), and fluid attenuated inversion recovery sequence (FLAIR), with the total scan time lasting less than ten minutes (Table 1). These BAT MRIs were evaluated for ischemia, hemorrhage, and other central nervous system pathologies by neuroradiologists and stroke neurologists at the time the scans were performed. This MRI is readily available at any time and may be obtained selectively for patients in whom a stroke diagnosis is in question. It is run in parallel to the IV-tPA selection process. A potential algorithm for the use of the BAT MRI is shown in Figure 1. The parameters for the sequences were identical to those used in routine complete MRI used to evaluate stroke patients. Statistical analysis was performed using $\mathrm{IBM}^{\oplus}$ SPSS $^{\circledast}$ Statistics Version 19 (Chicago, IL). Stroke risk factors in patients with and without BAT MRIs, among those with and without evidence of ischemia on BAT MRI, were compared using the t-test for continuous variables and Fisher's Exact Test for categorical variables with p-values less than 0.05 being considered as statistically significant. Variables not normally distributed are depicted as median (range), compared using Mann Whitney $\mathrm{U}$ test. The diagnosis at the time of the BAT MRI was compared to the discharge diagnosis, and the potential role of BAT MRI in IV-tPA administration decision in each case was evaluated.

\begin{tabular}{|l|c|}
\hline Type of Sequence & $\begin{array}{c}\text { Length of Sequence } \\
\text { (minutes:seconds) }\end{array}$ \\
\hline Localizer & $0: 09$ \\
\hline DWI & $1: 30$ \\
\hline T2 GRE & $1: 54$ \\
\hline Axial T2 & $2: 54$ \\
\hline Axial FLAIR & $3: 02$ \\
\hline Total & $\mathbf{8 : 4 9}$ \\
\hline
\end{tabular}

Table 1: BAT MRI Sequences.

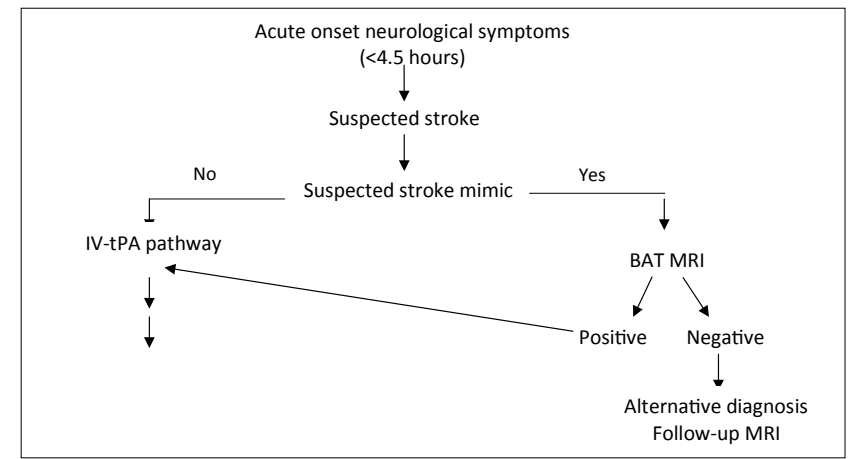

Figure 1: Algorithm for acute onset neurological symptoms.

This algorithm was used to treat patients in the study. It is also the general protocol used at Palmetto Health Richland.

\begin{tabular}{|c|c|c|c|}
\hline Stroke Risk Factors & $\begin{array}{l}\text { BAT MRI } \\
(n=82)\end{array}$ & $\begin{array}{c}\text { No BAT MRI } \\
(n=350)\end{array}$ & $\mathrm{p}$-value \\
\hline Age & $59 \pm 15$ & $65 \pm 16$ & 0.003 \\
\hline Male Gender & $45(55 \%)$ & $181(52 \%)$ & 0.61 \\
\hline Non-White Race & $44(54 \%)$ & $166(47 \%)$ & 0.31 \\
\hline Hypertension & $65(79 \%)$ & $273(78 \%)$ & 0.80 \\
\hline Diabetes & $24(29 \%)$ & $109(31 \%)$ & 0.74 \\
\hline Hypercholesterolemia & $31(38 \%)$ & $142(41 \%)$ & 0.65 \\
\hline Smoking & $36(44 \%)$ & $86(25 \%)$ & $<0.001$ \\
\hline $\begin{array}{l}\text { Coronary Artery } \\
\text { Disease }\end{array}$ & $13(16 \%)$ & $97(28 \%)$ & 0.03 \\
\hline Atrial Fibrillation & $11(13 \%)$ & $79(23 \%)$ & 0.07 \\
\hline History of TIA & $11(13 \%)$ & $35(10 \%)$ & 0.37 \\
\hline History of Stroke & $23(28 \%)$ & $91(26 \%)$ & 0.71 \\
\hline NIHSS§ & $4(2-36)$ & $5(1-35)$ & 0.31 \\
\hline IV-tPA administration & $6(7 \%)$ & $94(27 \%)$ & $<0.001$ \\
\hline
\end{tabular}

Normally distributed continuous values depicted as mean \pm standard deviation compared using a t-test. §Variables not normally distributed are depicted as median (range), compared using Mann Whitney $U$ test. Proportions depicted as (\%), categorical variables compared using Chi-square Test.

Table 2: Stroke risk factors present in acute stroke patients considered for and not considered for BAT MRI.

\section{Results}

In the 30 month period between July 2010 and December 2012, 432 patients (age range $19-99$ years; $48 \%$ male, $52 \%$ female; $50 \%$ black, $49 \%$ white, $1 \%$ others) presented within 4.5 hours of symptom onset with clinical features suggestive of AIS. Among these 432 patients, in 350 patients $(81 \%)$ the clinical diagnosis of stroke was certain and in the remaining $82(19 \%)$, the diagnosis of stroke was unclear and a stroke mimic was suspected. The patients receiving BAT MRI were younger (mean age \pm standard deviation $=59 \pm 15$ vs. $65 \pm 16 ; p=0.003$ ), more likely to be smokers ( $44 \%$ vs. $25 \%$; $\mathrm{p}<0.001$ ) and less likely ( $7 \%$ vs. $27 \%$; $<<0.001)$ to be selected for IV-tPA administration per NINDS ( $<3$ hours)/ECASS-III (3-4.5 hours) [17] criteria compared to those in whom a more definitive diagnosis of stroke precluded a BAT MRI (Table 2). The two groups were similar in gender and race composition, prevalence of diabetes, hypercholesterolemia, coronary artery disease, atrial fibrillation, history of stroke/TIA and NIHSS assessed on presentation.

In the 82 patients, BAT MRI was ordered after the initial noncontrast head CT was negative for hemorrhage or other acute processes (age range $19-93$ years; $45 \%$ male, 55\% female; 53\% $\%$ black, $46 \%$ white, $1 \%$ others). Of the 82 BAT MRIs, 25 were read as positive for acute 
Citation: Paolini S, Burdine J, Verenes M, Webster J, Faber T, et al. (2013) Rapid Short MRI Sequence Useful in Eliminating Stroke Mimics Among Acute Stroke Patients Considered for Intravenous Thrombolysis. J Neurol Disord 1: 137. doi:10.4172/2329-6895.1000137

Page 3 of 5

stroke (24 with acute ischemia and one positive for ischemia with hemorrhage). The patients with acute ischemia on BAT MRI were older (mean age \pm standard deviation $=67 \pm 14$ vs. $56 \pm 14 ; \mathrm{p}=0.003$ ), more likely to be males ( $72 \%$ vs. $47 \%$; $\mathrm{p}=0.04$ ), more likely to have hypercholesterolemia ( $60 \%$ vs. $28 \%$; $\mathrm{p}=0.01)$, have atrial fibrillation ( $32 \%$ vs. $5 \% ; \mathrm{p}=0.003$ ) and more likely to be selected for IV-tPA administration ( $24 \%$ vs. $0 \%$; $=0.001$ ) compared to those in whom no acute ischemia was detected by BAT MRI (Table 3 ). The two groups were similar in race composition, prevalence of hypertension, diabetes, smoking, coronary artery disease, history of stroke/ TIA and NIHSS assessed on presentation.

Among the 25 patients with evidence of AIS on MRI, 19 did not receive IV-tPA. In one of these 19 patients, a pontine hemorrhage not detected on CT was subsequently found on the BAT MRI, excluding the potentially harmful use of IV-tPA in the patient. Other patients were excluded from receiving IV-tPA for the following reasons: more than four and a half hours from symptom onset, positive stroke on MRI accompanied by rapidly improving stroke symptoms, or on anticoagulation with international normalization ratio (INR) $>1.6$ (discovered after rapid MRI performed as point of care INR was not available).

Six of the 25 patients with demonstrated AIS on MRI also met NINDS/ECASS-III criteria [18] and received IV-tPA within four and half hours of symptom onset. The average DTN for these patients was $92 \mathrm{~min} \pm 20 \mathrm{~min}$. The overall average DTN at the hospital over the same time period was $68 \pm 32$ minutes. Five out of the six IV-tPA cases had DTN time greater than 68 minutes. Overall the BAT MRI added an average of 24 minutes to the IV-tPA decision process. In the select group of patients, BAT MRI may have modestly increased the time to treatment; however, there were many factors at play in these cases. These factors are summarized in Table 4 . None of the patients became ineligible to receive IV-tPA because of the additional time added by obtaining the BAT MRI.

Of the 57 BAT MRIs negative for acute stroke, discharge diagnoses were as follows: 28 transient ischemic attacks (TIA), 2 MRI negative strokes, 14 conversion disorder/functional symptoms, 1 toxicmetabolic encephalopathy and the remaining 11 represented a myriad

\begin{tabular}{|l|c|c|c|}
\hline Stroke Risk Factors & $\begin{array}{c}\text { MRI: Acute } \\
\text { Ischemia } \\
\text { (n=25) }\end{array}$ & $\begin{array}{c}\text { MRI: No Acute } \\
\text { Ischemia } \\
\text { (n=57) }\end{array}$ & p-value \\
\hline Age & $\mathbf{6 7 \pm 1 4}$ & $\mathbf{5 6 \pm 1 4}$ & $\mathbf{0 . 0 0 3}$ \\
\hline Male Gender & $\mathbf{1 8 ( 7 2 \% )}$ & $\mathbf{2 7}(\mathbf{4 7 \% )}$ & $\mathbf{0 . 0 4}$ \\
\hline Non-White Race & $14(56 \%)$ & $30(53 \%)$ & 0.78 \\
\hline Hypertension & $22(88 \%)$ & $43(75 \%)$ & 0.20 \\
\hline Diabetes & $8(32 \%)$ & $16(28 \%)$ & 0.72 \\
\hline Hypercholesterolemia & $\mathbf{1 5 ( 6 0 \% )}$ & $\mathbf{1 6}(\mathbf{2 8 \% )}$ & $\mathbf{0 . 0 1}$ \\
\hline Smoking & $10(40 \%)$ & $26(46 \%)$ & 0.64 \\
\hline Coronary Artery Disease & $4(16 \%)$ & $9(16 \%)$ & $>0.99$ \\
\hline Atrial Fibrillation† & $\mathbf{8 ( 3 2 \% )}$ & $\mathbf{3 ( 5 \% )}$ & $\mathbf{0 . 0 0 3}$ \\
\hline History of TIA† & $2(8 \%)$ & $9(16 \%)$ & 0.49 \\
\hline History of Stroke & $7(28 \%)$ & $16(28 \%)$ & $>0.99$ \\
\hline NIHSS§ & $4(2-26)$ & $4(2-20)$ & 0.57 \\
\hline IV-tPA administration† & $\mathbf{6 ( 2 4 \% )}$ & $\mathbf{0}$ & $\mathbf{0 . 0 0 1}$ \\
\hline
\end{tabular}

Normally distributed continuous values depicted as mean \pm standard deviation, compared using a t-test. §Variables not normally distributed are depicted as median (range), compared using Mann Whitney $U$ test. Proportions depicted as (\%), categorical variables compared using Chi-square test, except when appropriate (cell $<5)$, † Fisher's Exact Test was used.

Table 3: Stroke risk factors present in patients with acute ischemia and without acute ischemia on BAT MRI.

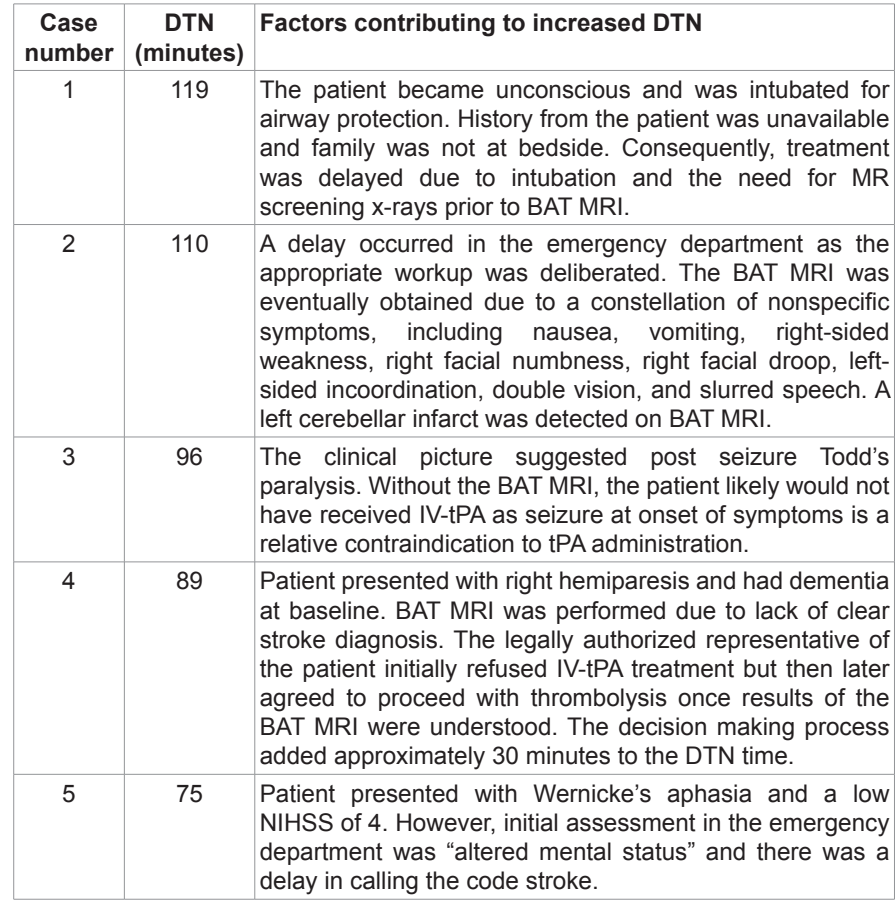

Table 4: Summary reason for delay in DTN in BAT MRI subjects receiving IV-tPA

\begin{tabular}{|l|l|c|}
\hline & Discharge Diagnosis & $\begin{array}{c}\text { Number of } \\
\text { Patients (N=82) }\end{array}$ \\
\hline MRI: Ischemia Positive & Acute Ischemia & $24(29 \%)$ \\
\hline & Ischemia + Hemorrhage & $1(1.2 \%)$ \\
\hline MRI: Ischemia Negative & Transient Ischemic Attack & $28(34 \%)$ \\
\hline & MRI-Negative Stroke & $2(2.4 \%)$ \\
\hline & Hemorrhage & $2(2.4 \%)$ \\
\hline & Conversion Disorder/ Functional & $14(17 \%)$ \\
\hline & Toxic-Metabolic Encephalopathy & $1(1.2 \%)$ \\
\hline & Vertigo & $1(1.2 \%)$ \\
\hline & Acute Respiratory Failure & $1(1.2 \%)$ \\
\hline & Drug Seeking Behavior & $1(1.2 \%)$ \\
\hline & Alcohol intoxication & $1(1.2 \%)$ \\
\hline & Complex Migraine & $1(1.2 \%)$ \\
\hline & Seizure & $1(1.2 \%)$ \\
\hline & Bell's Palsy & $1(1.2 \%)$ \\
\hline & Transverse Myelitis & $1(1.2 \%)$ \\
\hline & Neoplasm & $1(1.2 \%)$ \\
\hline & Trauma & $1(1.2 \%)$ \\
\hline & & \\
\hline
\end{tabular}

Table 5: Discharge Diagnosis. Percent (\%) designates proportion of all diagnoses.

of other illnesses (Table 5). Of the two MRI negative strokes, one patient had persistent minor symptoms and the follow-up complete MRI scan was negative and in one patient the follow-up MRI was positive for ischemia. Eight out of the fourteen patients diagnosed with functional symptoms had a previous psychiatric diagnosis. In all of these patients the psychiatric/functional diagnoses were confirmed through a formal psychiatry consultation.

\section{Discussion}

The use of MRI in acute stroke has been a subject of debate for over a decade [19]. Previous studies using various MRI sequence combinations and different stroke treatment protocols have demonstrated the feasibility of rapid MRI in patients presenting with acute stroke-like symptoms [14-17]. These studies have used MRI 
Citation: Paolini S, Burdine J, Verenes M, Webster J, Faber T, et al. (2013) Rapid Short MRI Sequence Useful in Eliminating Stroke Mimics Among Acute Stroke Patients Considered for Intravenous Thrombolysis. J Neurol Disord 1: 137. doi:10.4172/2329-6895.1000137

Page 4 of 5

obtained $<6$ hours from symptom onset to assess diffusion-perfusion mismatch to detect salvageable brain, vascular imaging using MR angiography to assess candidacy for acute interventional stroke treatment, as well as gradient echo imaging to rule out intracranial hemorrhage. However, three recent studies have shown the lack of utility of MR diffusion-perfusion mismatch to assess candidacy for IV-tPA beyond the 3 hour time frame [20]. Based on these studies, the recent acute stroke guidelines dissuade the use of additional imaging that may delay treatment with IV thrombolysis [21]. Additionally, the MR-RESCUE study showed the lack of utility of MR diffusionperfusion mismatch to assess candidacy for interventional stroke management [22]. Consistent with these evidence-based guidelines, we developed a short, rapid BAT MRI to confirm AIS, exclude stroke mimics, and assess candidacy for IV-tPA in select patients in whom the diagnosis is not clear. In the prior rapid MRI studies, the image acquisition time ranged from five minutes [16] to 12 minutes [17], and included magnetic resonance angiography (MRA) and perfusion weighted imaging [15-17]. The present study image acquisition time was 8 minutes 49 seconds (Table 1). This included DWI, T2 GRE, T2 axial, and FLAIR and eliminated MRA and perfusion weighted imaging. By using the BAT MRI algorithm and protocol, the DTN time for IV-tPA administration was modestly increased in a select subgroup of patients presenting with suspect stroke symptoms less than four and half hours from symptom onset.

The present study, unlike previous studies, focused on the capacity of the rapid BAT MRI to exclude stroke mimics. Heidenreich et al. found that MRI evaluation altered the treatment course in $26 \%$ of patients [17]. In this study, in a select group of patients, the BAT MRI likely altered treatment in $70 \%$ of patients (Table 5), as it aided in the diagnosis of TIAs and helped exclude stroke mimics in the majority of these patients. Additionally in one patient, BAT MRI detected a pontine hemorrhage not detected on $\mathrm{CT}$ and prevented this patient from potential harm from receiving thrombolysis therapy.

There are potential limitations of this study. These include physician bias in determining which patients receive the BAT MRI, the modest sample size of the study, and the retrospective nature of the study. The selection bias is suggested by the fact that the patients receiving BAT MRI were younger, more likely to be smokers and less likely to be selected for IV-tPA administration compared to those in whom a more definitive diagnosis of stroke precluded a BAT MRI (Table 2). There is also the possibility that the BAT MRI may miss AIS, as evidenced by the two MRI negative strokes. One of these strokes was found on follow-up imaging the next day. Overall, the BAT MRI was able to detect evidence of ischemia in $30 \%$ of patients in whom stroke diagnosis was not clear. The patients with acute ischemia on BAT MRI were older, more likely to be males, and more likely to have hypercholesterolemia and atrial fibrillation (Table 3). These clinical features increased the odds of MRI evidence of ischemia and the patient receiving IV t-PA.

Additionally, there is the concern that the MRI, though rapid, may delay DTN time for patients selected to receive IV-tPA (Table 4). Though the average DTN time was high in several of the cases in this study, the patients would not have been treated without evidence of ischemia on the BAT MRI. Treatment occurred within 4.5 hours of symptom onset, and it has been demonstrated that treatment in this time window significantly improves clinical outcomes [23]. Additionally, it is unlikely that the increased DTN time in these cases can be attributed only to the BAT MRI. Although there is a basic algorithm for use of the BAT MRI (Figure 1), it may be necessary to use process improvement strategies to effectively and efficiently incorporate the BAT MRI into the t-PA pathway and shorten the DTN time. Further studies are needed to determine the cost-effectiveness of an MR based thrombolysis protocol in select patients with suspected stroke.

\section{Conclusion}

In patients with acute stroke-like symptoms, BAT MRI may be used to confirm acute ischemic stroke, exclude stroke mimics, and assess candidacy for IV-tPA. While BAT MRI may be useful in select patients, current guidelines emphasize the use of $\mathrm{CT}$ alone to avoid delay in the IV-tPA decision making. Hence caution is advised regarding ordering an ultrashort MRI scan that may potentially delay or exclude appropriate patients from receiving IV-tPA. Further studies are needed to confirm our findings. Based on this study, implementation of BAT MRI may assist in preventing select patients from inadvertently receiving treatment for stroke misdiagnosis.

\section{Funding}

Dr. Paolini was supported for this proposal by the AHA Student Scholarship in Cerebrovascular Disease and Stroke, and Dr. Sen is supported by NINDS Grant number 1R01NS062754.

\section{References}

1. Kwiatkowski TG, Libman RB, Frankel M, Tilley BC, Morgenstern LB et al (1999) Effects of tissue plasminogen activator for acute ischemic stroke at one year. National Institute of Neurological Disorders and Stroke Recombinant Tissue Plasminogen Activator Stroke Study Group. N Engl J Med 340: 17811787.

2. (1995) Tissue plasminogen activator for acute ischemic stroke. The National Institute of Neurological Disorders and Stroke rt-PA Stroke Study Group. N Engl J Med 333: 1581-1587.

3. Del Zoppo GJ, Saver JL, Jauch EC, Adams HP, Jr. (2009) Expansion of the time window for treatment of acute ischemic stroke with intravenous tissue plasminogen activator: a science advisory from the American Heart Association/American Stroke Association. Stroke 40: 2945-2948.

4. Lev MH (2003) CT versus MR for acute stroke imaging: is the "obvious" choice necessarily the correct one? AJNR Am J Neuroradiol 24: 1930-1931.

5. Chernyshev OY, Martin-Schild S, Albright KC, Barreto A, Misra V, et al. (2010) Safety of tPA in stroke mimics and neuroimaging-negative cerebral ischemia. Neurology 74: 1340-1345.

6. Sen S, Oppenheimer S (2008) Bedside assessment of stroke and stroke mimics. Annals of Indian Academic Neurology 11: S4-S11.

7. Winkler DT, Fluri F, Fuhr P, Wetzel SG, Lyrer PA, et al. (2009) Thrombolysis in stroke mimics: frequency, clinical characteristics, and outcome. Stroke 40 1522-1525.

8. Johnston SC (2010) The economic case for new stroke thrombolytics. Stroke 41: S59-62.

9. Mar J, Begiristain JM, Arrazola A (2005) Cost-effectiveness analysis of thrombolytic treatment for stroke. Cerebrovasc Dis 20: 193-200.

10. Tung CE, Win SS, Lansberg MG (2011) Cost-effectiveness of tissue-type plasminogen activator in the 3 - to 4 .5-hour time window for acute ischemic stroke. Stroke 42: 2257-2262.

11. Zinkstok SM, Engelter ST, Gensicke H, Lyrer PA, Ringleb PA, et al. (2013) Safety of thrombolysis in stroke mimics: results from a multicenter cohort study. Stroke 44: 1080-1084.

12. Chalela JA, Kidwell CS, Nentwich LM, Luby M, Butman JA, et al. (2007) Magnetic resonance imaging and computed tomography in emergency assessment of patients with suspected acute stroke: a prospective comparison. Lancet 369: 293-298.

13. Saver JL, Levine SR (2010) Alteplase for ischaemic stroke--much sooner is much better. Lancet 375: 1667-1668.

14. Sunshine JL, Tarr RW, Lanzieri CF, Landis DM, Selman WR, et al. (1999) 
Citation: Paolini S, Burdine J, Verenes M, Webster J, Faber T, et al. (2013) Rapid Short MRI Sequence Useful in Eliminating Stroke Mimics Among Acute Stroke Patients Considered for Intravenous Thrombolysis. J Neurol Disord 1: 137. doi:10.4172ł2329-6895.1000137

Page 5 of 5

Hyperacute stroke: ultrafast MR imaging to triage patients prior to therapy. Radiology 212: 325-332.

15. Schellinger PD, Jansen O, Fiebach JB, Pohlers O, Ryssel H, et al. (2000) Feasibility and practicality of MR imaging of stroke in the management of hyperacute cerebral ischemia. AJNR Am J Neuroradiol 21: 1184-1189.

16. U-King-Im JM, Trivedi RA, Graves MJ, Harkness K, Eales H, et al. (2005) Utility of an ultrafast magnetic resonance imaging protocol in recent and semi-recent strokes. J Neurol Neurosurg Psychiatry 76: 1002-1005.

17. Heidenreich JO, Hsu D, Wang G, Jesberger JA, Tarr RW, et al. (2008) Magnetic resonance imaging results can affect therapy decisions in hyperacute stroke care. Acta Radiol 49: 550-557.

18. Hemmen TM, Rapp KS, Emond JA, Raman R, Lyden PD (2010) Analysis of the National Institute of Neurological Disorders and Stroke tissue plasminogen activator studies following European Cooperative Acute Stroke Study III patient selection criteria. J Stroke Cerebrovasc Dis 19: 290-293.
19. Oliveira-Filho J, Koroshetz WJ (2000) Magnetic resonance imaging in acute stroke: clinical perspective. Top Magn Reson Imaging 11: 246-258.

20. Wechsler LR (2011) Imaging evaluation of acute ischemic stroke. Stroke 42 S12-S15.

21. Jauch EC, Saver JL, Adams HP, Bruno A, Connors JJ, et al. (2013) Guidelines for the Early Management of Patients With Acute Ischemic Stroke: A Guideline for Healthcare Professionals From the American Heart Association/American Stroke Association. Stroke. 3: 870-947.

22. Kidwell CS, Jahan R, Gornbein J, Alger JR, Nenov V, et al. (2013) A trial of imaging selection and endovascular treatment for ischemic stroke. $\mathrm{N}$ Engl J Med 368: 914-923.

23. Hacke W, Kaste M, Bluhmki E, Brozman M, Dávalos A, et al. (2008) Thrombolysis with alteplase 3 to 4.5 hours after acute ischemic stroke. N Engl J Med 359: 1317-1329. 